\title{
Free-standing films from chirality-controlled carbon nanotubes
}

\author{
Dawid Janas $^{\mathrm{a}, \mathrm{b},{ }^{*}, \text { Monika Rdest }}{ }^{\mathrm{a}}$, Krzysztof K.K. Koziol ${ }^{\mathrm{a}}$ \\ a Department of Materials Science and Metallurgy, University of Cambridge, 27 Charles \\ Babbage Rd, CB3 OFS Cambridge, United Kingdom \\ b Department of Chemistry, Silesian University of Technology, B. Krzywoustego 4, 44-100 \\ Gliwice, Poland
}

\begin{abstract}
Development of a method of formation of chiral free-standing films from carbon nanotubes (CNTs), which circumvents the limitations of the established techniques, is presented. CNT macroassemblies of any size and shape can be made from desired CNT feed including those synthesized or sorted with the objective of chirality control. Free-standing CNT films were created from various starting CNT materials the most interesting being of predominantly $(6,5)$ and $(7,6)$ chirality. The technique offers the advantages of technical simplicity, scalability and non-destructive nature representing an important step toward the research, development and implementation of monochiral single-wall CNT tangible objects.
\end{abstract}

\footnotetext{
${ }^{*}$ Corresponding author. Tel: + 4832 2372958. E-mail address: dawid.janas@ polsl.pl (D. Janas).
} 


\section{Introduction}

Despite remarkable properties of individual carbon nanotubes (CNTs), a problem arises when one tries to extend these features onto their macroscopic assembly. There is a selection of methods how CNT macroassemblies such as films[1] or fibers [2] can be made. In general, the techniques can be divided between liquid medium-based and solid processing. The former case involves dispersing particular CNT material in a solvent, often with the help of stabilizing agent such as surfactant $[3,4]$ or pre-functionalization of the CNTs $[5,6]$. The CNT dispersion is then drawn to yield CNT fibers [7-9] or deposited on a planar surface to produce CNT films [10-12]. The most significant disadvantage of wet-processes is the need to functionalize CNTs or use indispensable binding agent, which deteriorate the properties of the CNTs. What regards the latter approach, the dry-processing such as array-drawing $[13,14]$ or direct-spinning $[15,16]$ do not involve functionalization or a binding compound, but the produced CNT macrostructure is at best only enriched with the CNTs of particular type. Having the ability to combine these two worlds and produce binder-free CNT macroassemblies of a desired type has remained a challenge to this day.

Since the influential Iijima's report on the formation of carbon nanotubes (CNTs) in 1991 [17], the research community has put continuous efforts in utilizing impressive CNT properties. Individual CNTs have shown remarkable performance in terms of electrical [18-20], thermal [21, $22]$, mechanical $[23,24]$, optical $[25,26]$ and other attributes. It is not uncommon for individual CNTs to outperform classical bulk-type materials by orders of magnitude on the nanoscale e.g. by having three orders of magnitude higher critical current density [18] or an order of magnitude higher thermal conductivity [22] than copper. However, a problem arises when one tries to extend these properties beyond an isolated CNT. A collection of CNTs is also composed of 
junctions and voids which deteriorate the performance. Compaction of the material and thus good contact between individual CNTs is crucial for appropriate electrical/thermal conductivity, stress transfer and other attributes. Moreover, the constituting CNTs should ideally be defect-free and preferably of a particular chirality.

There is a selection of methods how CNT macroassemblies such as films [1] or fibers [2] can be made. In general, the techniques can be divided between liquid medium-based and solid processing. The former case involves dispersing the CNT material in a solvent, often with the help of stabilizing agent such as surfactant $[3,4]$ or pre-functionalization of the CNTs $[5,6]$. The CNT dispersion is then drawn to yield CNT fibers [7-9], filtered [27-29] or deposited on a planar surface to produce CNT films [10-12]. The most significant disadvantages of wet-processes is the need to functionalize CNTs or use indispensable binding agent, which deteriorate the properties of the CNTs. What regards the dry-processing methods such as array-drawing [13, 14] or direct-spinning $[15,30]$ do not involve functionalization nor a binding compound, but the produced CNT macrostructure is at best only enriched with the CNTs of particular type. Having the ability to combine these two worlds and produce binder-free CNT macroassemblies of a desired type has remained a challenge to this day.

In this report we present an universal and rapid method of production of such self-supported CNT films of e.g. predominantly monochiral nature. Carbon nanostructures are dispersed in a solvent with a specially designed agent, which stabilizes the dispersion, provides scaffolding for CNT film formation and can be easily removed from such CNT film leaving no residue. In this process, as formed carbon paint is deposited onto a support, on which it forms a robust thin film, which can be peeled off from the surface. Removal of binder by thermal annealing yields a freestanding carbon nanostructure macroassembly of arbitrary size and shape. As compared with 
most methods to produce CNT macroassemblies, the dispersing agent left no trace in the material after the whole processing. Moreover, we eliminated the need for difficult to scale-up filtration step, what is the case with most buckypaper manufacture methods. The technique opens new routes towards creation of high-purity carbon macrostructures of predetermined chemical composition, microstructure or chirality in the long term as any CNT type can be used as feed to create macroassemblies this way.

\section{Experimental}

\subsection{Materials}

Four types of CNTs were used in this study. NC7000 CNTs were purchased from Nanocyl, whereas CNT carpets were synthesized in house from CVD of toluene and ferrocene at $760^{\circ} \mathrm{C}$ under argon by a typical CVD procedure. We have also employed chirality-enriched $(7,6)$ and $(6,5)$ CNTs from SouthWest NanoTechnologies. To disperse nanocarbon material in a solvent and improve the integrity of the film, ethyl cellulose was employed as binder (in all the ratios of binder to CNTs we have tested, 1:1 appears to be the optimum for the dispersion quality. It is possible to manufacture the CNT film with the ratio of binder to CNTs being lower, but then the nanocarbon does not disperse easily in short time).

\subsection{Preparation of dispersion}

Binder and selected CNT powder were added to a solvent carrier (iso-propanol) and the mixture was sonicated for $15 \mathrm{~min}$ (Branson $450 \mathrm{CE}$ sonicator) until the dispersion became homogeneous and no lumps of the material could be seen.

\subsection{Deposition}

The dispersion was then deposited onto a Kapton foil by spray-coating inside a custom-built deposition chamber ( 3 bar pressure was employed to achieve a fine mist of material) to create a 
robust thin film. The deposition was stopped when equal volumes of dispersions for all the materials have been exhausted.

\subsection{Flash annealing}

As-prepared samples were finally ignited with a blowtorch, which rapidly annealed the sample from binder in a couple of seconds. The whole process is illustrated in Fig. 1.

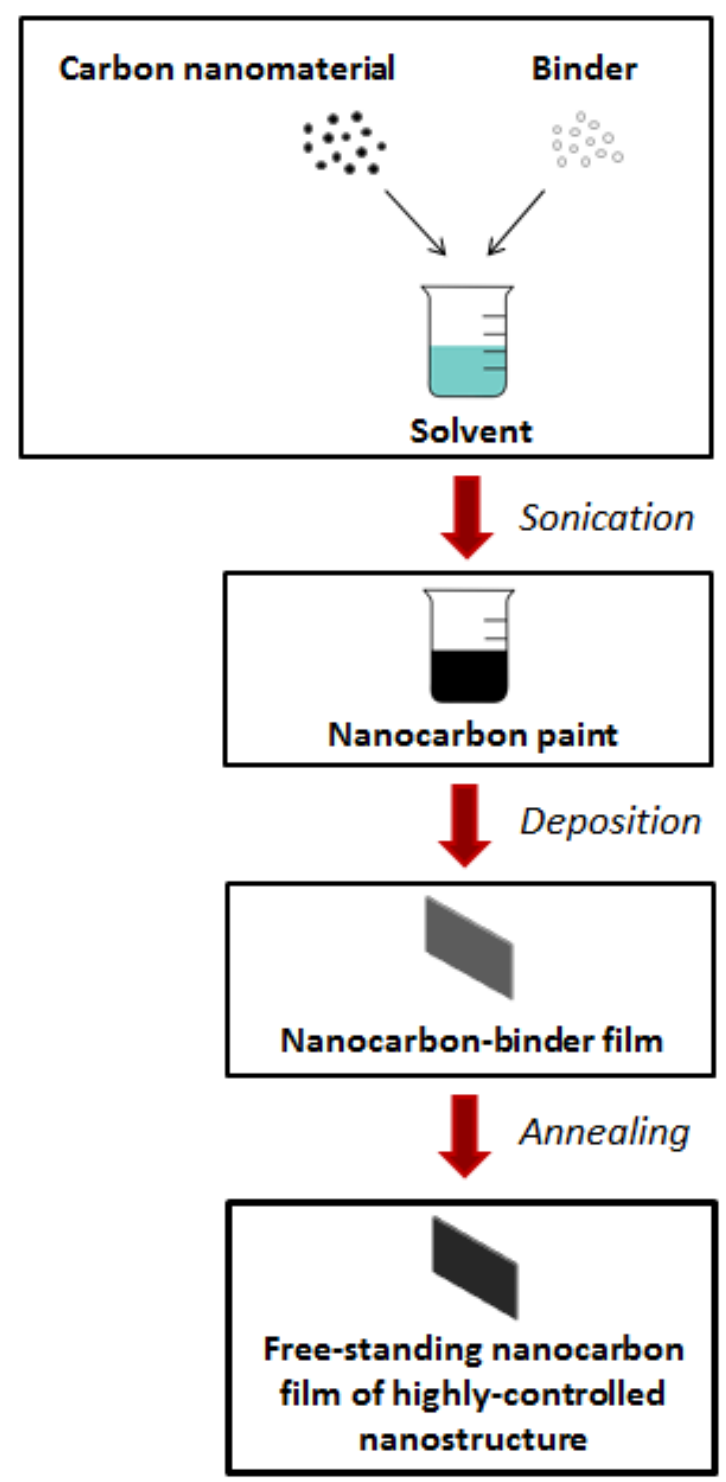

Fig. 1. The manufacture process. 


\subsection{Characterization}

Scanning and Transmission Electron Microscopy (SEM, FEI Nova NanoSEM; TEM, Tecnai Osiris FEGTEM) were used to probe the structure and quality of CNTs films on micro- and nanoscale.

The optical absorption spectra are acquired in a Varian Cary 5000 UV-Vis-NIR spectrophotometer at $780 \mathrm{~nm}$. Measurements were carried out in the range 350-1350 nm, which is sufficient to cover the first and second excitonic transitions.

Raman spectroscopy (Renishaw inVia, $\lambda=633 \mathrm{~nm}$ laser) gauged chemistry of the samples' surface by measuring the ratio of intensity of defect induced band (D) to the band of vibrations of graphitic structures $(\mathrm{G})$. We collected ten accumulations in the static recording mode from 1150 to $1800 \mathrm{~cm}^{-1}$.

Energy-Dispersive X-ray spectroscopy (EDX, Bruker Quantax coupled with Nova NanoSEM) detected the elemental composition at $10 \mathrm{keV}$ emission voltage. Spectral composition was always normalized to the content of carbon.

Thermogravimetric analysis (TGA, Mettler Toledo TGA/DSC system) measured changes in chemical and physical properties with temperature. The samples were heated to $1000^{\circ} \mathrm{C}$ at $10^{\circ} \mathrm{C}$

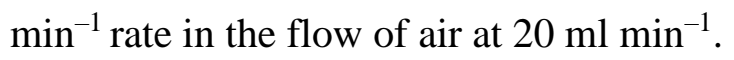

\subsection{Electrical testing of specimens}

The relation between temperature and resistance was measured by making stepwise increase in bias voltage and recording average temperature of the film by a thermal camera (Flir T440) until the film was burned across in a scission-like fashion. To compare the trends among samples, measured resistance was normalized to the initial resistance measured at room temperature. Moreover, electrical conductivity was measured by taking two-point resistance and cross- 
sectional area of five samples each. SEM imaging gave thickness of the films, and thus their cross-sectional area. $10 \mathrm{~mm} \times 40 \mathrm{~mm}$ specimens were employed for testing.

\subsection{Mechanical testing of specimens}

To evaluate the tensile strength of the films we used a universal testing machine (Instron). The gauge length and loading rate were set to $15 \mathrm{~mm}$ and $1 \mathrm{~mm} \mathrm{~min}^{-1}$, respectively. 5 specimens were tested for each sample type.

Durability and mechanical integrity of the CNT films was also gauged by a previously reported method [31]. In brief, the specimens $(10 \mathrm{~mm}$ x $60 \mathrm{~mm})$ were subjected to Three-point bending (Dynamic Mechanical Analysis, TA Instruments Q800DMA) during which electrical resistance was monitored by an on-line multimeter connected to a PC (Precision Gold, N56FU). The selected measurement parameters were: 1 bending cycle per minute $(1 / 60 \mathrm{~Hz}), 1 \mathrm{~mm}$ amplitude,

1440 cycles $(24 \mathrm{~h})$. The variation of electrical resistance as a function of the number of bending cycles gives information about the mechanical strength of the CNT films. The measured resistance of each tested sample was normalized to the initial resistance to enable comparison between samples. 


\section{Results and discussion}

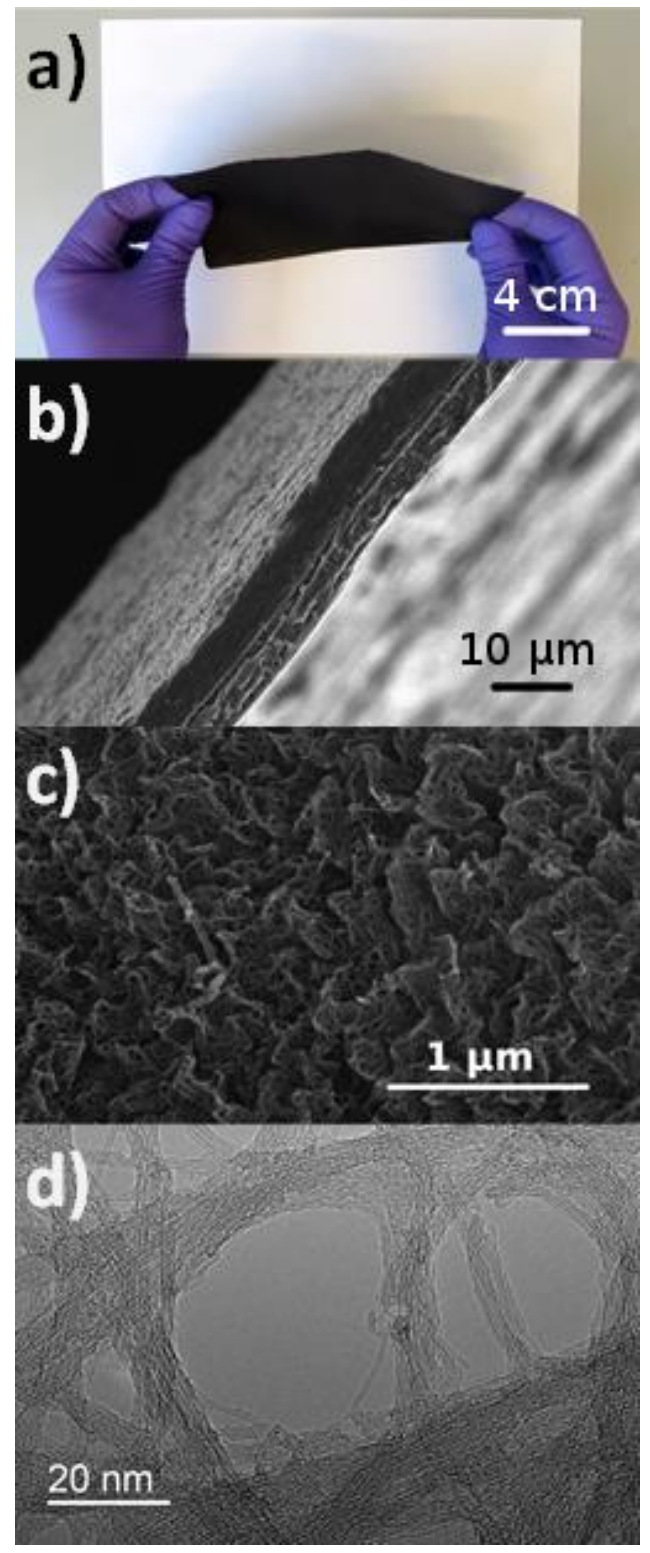

Fig. 2. Free-standing CNT film made from predominantly (6,5) CNTs. a) Visual appearance. b) $10 \mu \mathrm{m}$ thick cross-section. c) SEM micrograph. d) TEM micrograph

The films are mechanically stable, durable and very flexible. They can be made in any size and shape with uniform thickness ranging from few to hundred microns (Fig. 2). Repeated high bend angle manipulation does not cause any deterioration to the structure or properties of the material. 
We also subjected them repeatedly to ultra-low (immersion into liquid nitrogen) or elevated temperatures (keeping in a laboratory oven at $400^{\circ} \mathrm{C}$ for prolonged time), which did not impair their mechanical integrity. Most importantly, these sheets can be made using any CNT feed with most interesting being that of monochiral architecture. We selected chirality-prevalent $(6,5)$ CNTs as an example for the main text, but we have also tested other types of CNTs of different microstructure and chemical composition. To prove the universal nature of the proposed method, results from the following materials are also described in the Supplementary Information file: industrial-grade NC7000 product, vertically-aligned CNT carpet and chirality-prevalent $(7,6)$ CNT powder (Fig. S3-S5).

Investigation by electron microscopy confirmed $(6,5)$ to be single-wall and of small diameter $0.81 \pm 0.05 \mathrm{~nm}$ (Fig. 2d,e). Furthermore, optical absorption spectra also indicated that at least 50\% of the content of the sample is of the indicated chirality (Fig. S1) with the sample being of very high purity based on low $\mathrm{I}_{\mathrm{D}} / \mathrm{I}_{\mathrm{G}}$ ratio of 0.065 (Fig. 3a). The emergence of characteristic $\mathrm{M}$ overtone and splitting of $G$ band into two components $\mathrm{G}^{-}$and $\mathrm{G}^{+}$prove its semi-conductive nature [32], as expected. 

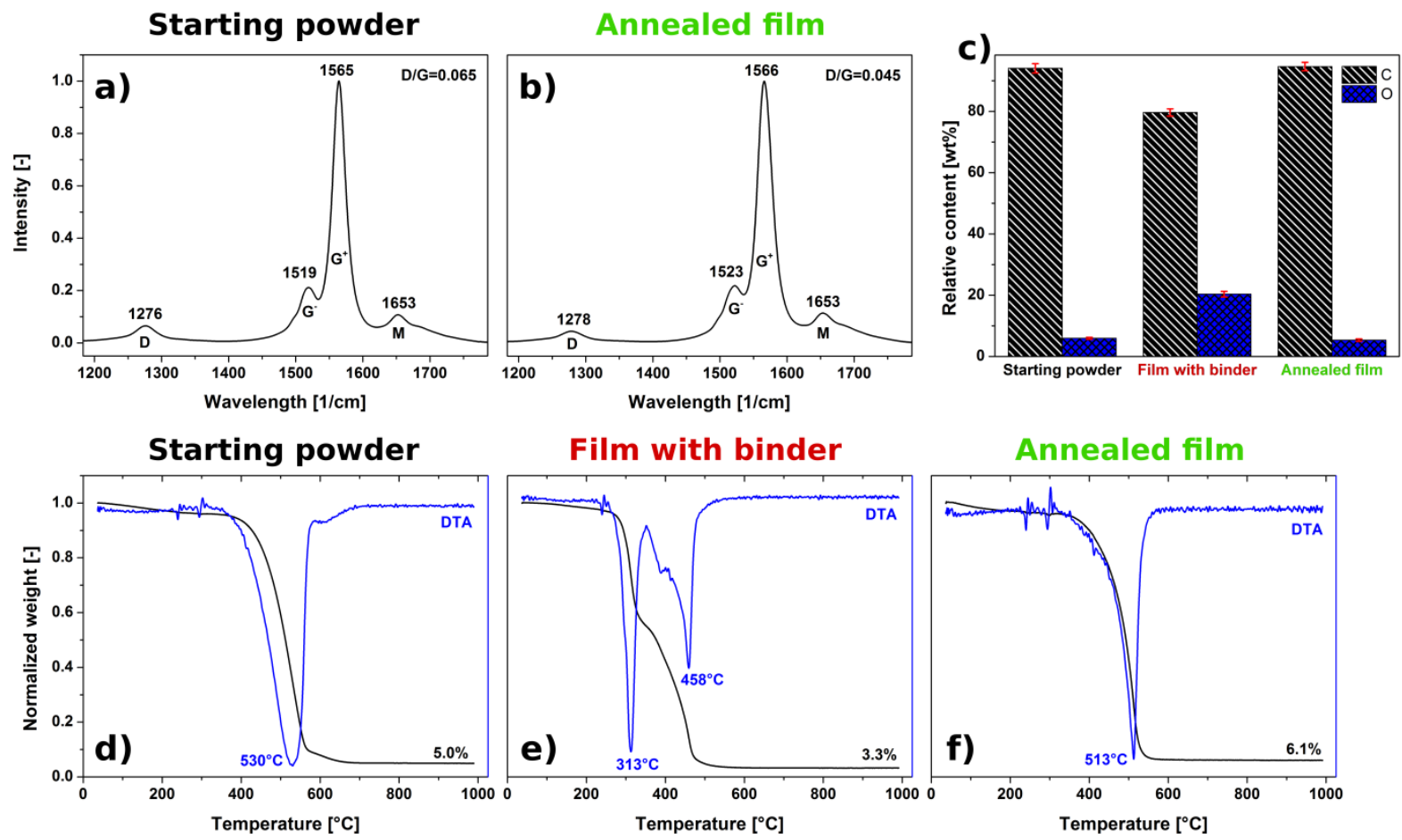

Fig. 3. The influence of processing on the properties and composition of the $(6,5)$ material. a,b) Raman spectra of the starting powder and annealed film. c) Oxygen content of the starting powder, CNT film with binder and annealed film as measured by EDX. d-f) Thermograms of the starting powder, CNT film with binder and annealed film.

Processing of the powder into a film via sonication and flash thermal annealing did not affect the composition of the material. The $\mathrm{I}_{\mathrm{D}} / \mathrm{I}_{\mathrm{G}}$ ratio actually decreased slightly down to 0.045 (Fig. 3b) possibly due to the removal of the most defected species, which gave major contribution to the disorder band D. Because of the presence of high background in the Raman spectrum of the CNT film with binder, it is not shown.

Next, chemical composition as measured by EDX (Fig. 3c) showed that the increase in oxygen content as a result of ethyl cellulose addition is fully reversible and rapid thermal removal of binder restores the initial oxygen content. We found that, owing to the flame retardant properties 
of the CNTs [33], they can sustain short exposure to high temperature without oxidation. In our case no more than a couple of seconds was required to carry out the annealing. It is encouraging to see that even the least thermally stable type of the CNTs having just a single-wall of carbon atoms survived this step without harm.

Lastly, we explored the influence of processing on the thermal characteristics of the material by means of thermogravimetric analysis (TGA). Fig. 2d shows thermal decomposition of the starting $(6,5)$ material, wherein we recorded the maximum rate of decomposition temperature to be $530^{\circ} \mathrm{C}$, which is in accordance with the values measured for single-wall CNTs. Introduction of binder (with its two characteristic peaks of decomposition at about $350^{\circ} \mathrm{C}$ and $450^{\circ} \mathrm{C}$, Fig. 4) to the starting CNT material, was correctly reflected in the observed thermogram (Fig. 2e).

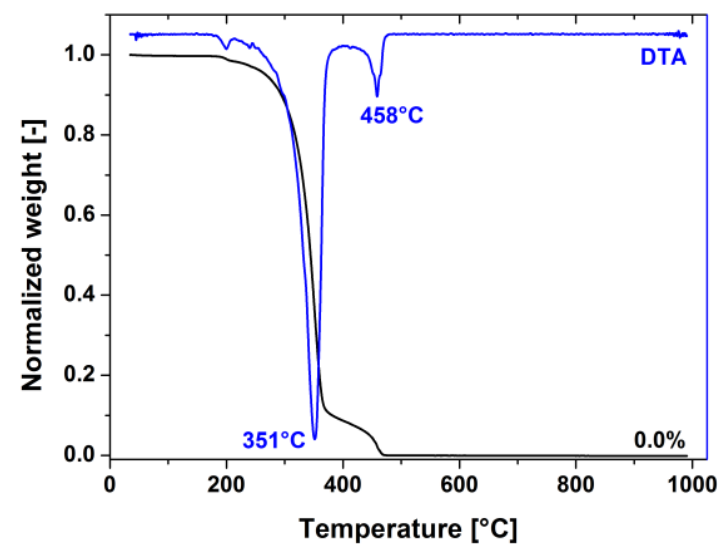

Fig. 4. Thermogram of ethyl cellulose.

Because single-wall CNTs such as $(6,5)$ are much less thermally stable than double-wall and multi-wall CNTs [34], binder oxidation may coincide with their oxidation regime as shown in the plots. That is why it is very important to use rapid methods of binder removal such as flash annealing, which we developed for this process to leave the inherent properties of the CNTs 
intact. Another important feature of the ethyl cellulose binder is the lack of residue left after its thermal decomposition (Fig. 4). In a common scenario, once the dispersing agent (typically longtail organic surfactant) is combined with CNTs the repercussions of interaction become permanent due to the fact that one cannot fully remove its traces. Here however, the ashless character of ethyl cellulose is ideal for this purpose. The thermogram of the annealed film (Fig. 3f) very much resembles that of the starting material. The same is true for the material microstructure as observed by SEM images (Fig. S2). In light of all these facts we can conclude that ethyl cellulose is a very suitable, non-intrusive vector to create such films.
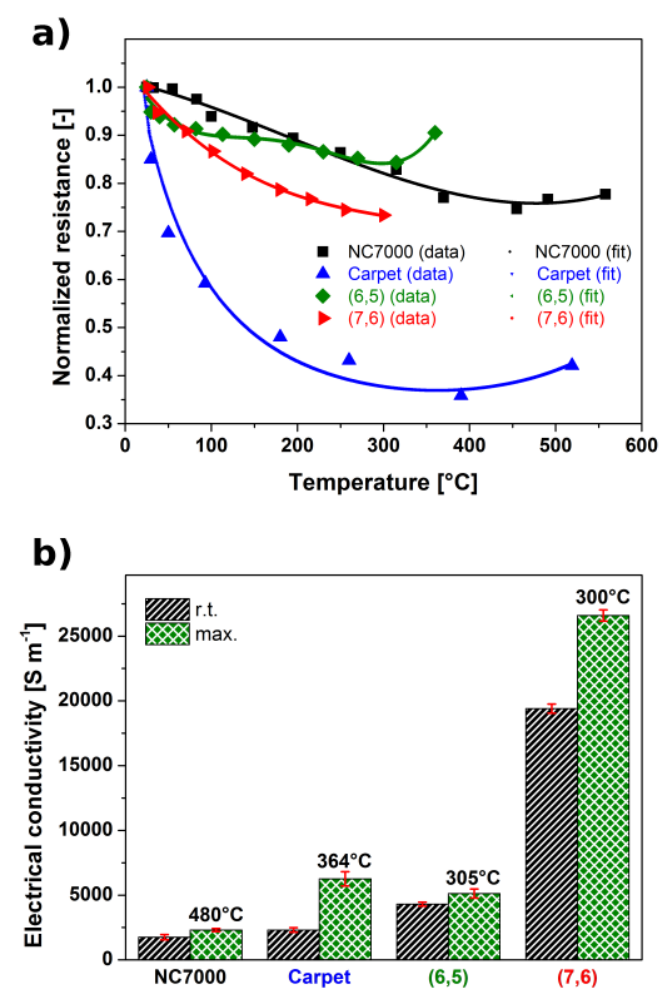

Fig. 5. Electrical properties. a) relation between resistance and temperature. b) electrical conductivity measured at room temperature and highest measured electrical conductivity at elevated temperatures (upturn points) of the corresponding CNT films. 
Because the structure of CNTs at the nanoscale plays a predominant role in their electrical properties, we wanted to evaluate how they change with the nature of CNTs chosen for film manufacture. Firstly, we measured how electrical resistance of such macroassemblies varies with temperature for all the materials produced in this work (Fig. 5a).

In all of the cases we saw a decrease of resistance with temperature, what was most evident in the case of vertically-aligned carpet-based material, which experienced more than $65 \%$ reduction. We have also noted a decrease of resistance with temperature for $(6,5)$ and $(7,6)$ samples, which was expected due to their inherent semiconductive nature.

Finally, as can be seen in the plot, the film made from NC7000 CNTs was also of predominantly semiconductive character. The upturn points show temperatures above which oxidation comes into play and start to slowly decompose the samples until electrical circuit is broken. Both NC7000 and carpet, which are based on multi-wall CNTs, were found much more thermally stable than single-wall CNT-based $(6,5)$ and $(7,6)$, what is in accordance with results reported from TGA earlier in text

Having the ability to create macroassemblies from these CNT materials enabled us also to evaluate their electrical conductivity (Fig. 5b). The highest values were obtained in the case of CNT films of predominant single-chirality, wherein the leader was found to be $(7,6)$ with 19,500 $\mathrm{S} / \mathrm{m}$ at room temperature. That is probably because single-wall CNTs have higher conductivity than multi-wall CNTs. Typical bundles in single-wall CNTs help with the electron transfer and make the establishment of percolation pathways easier. Lastly, we have also measured what the electrical conductivity at elevated temperatures was due to decrease of resistance with temperature. Despite the fact that carpet-based CNT film showed the most significant relative 
decrease of resistance by about $65 \%$, the initial resistance of $(7,6)$ was much lower and it was once again the most conductive samples among the ones tested. At the temperature of minimum resistance being $300^{\circ} \mathrm{C}$ (upturn point), $(7,6)$ reached $26,600 \mathrm{~S} / \mathrm{m}$.
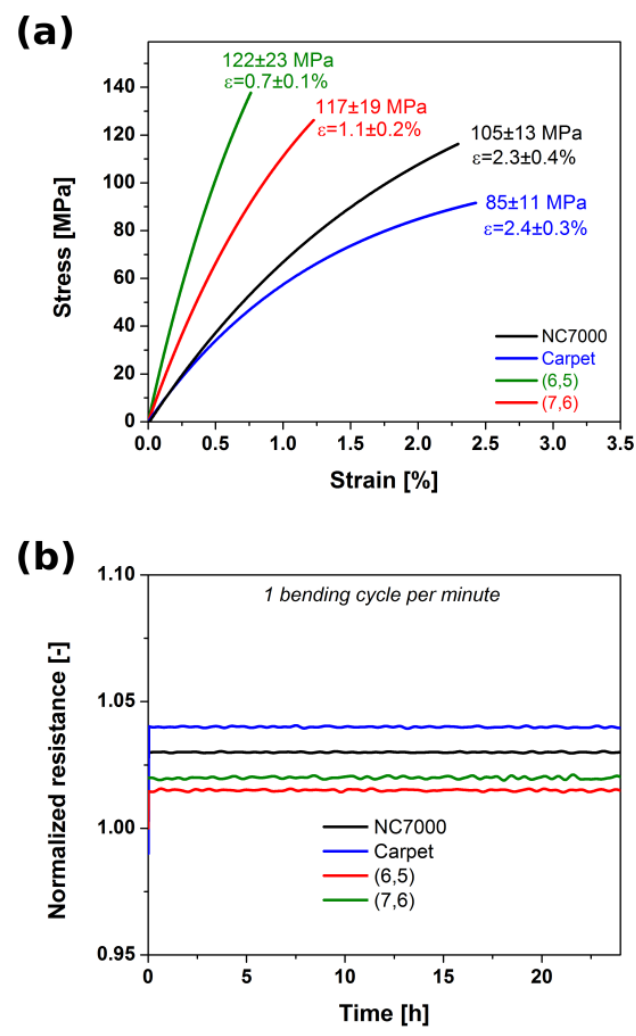

Fig. 5. (a) Stress-strain curves and (b) Electromechanical properties: stability of electrical resistance of the CNT films subjected to repeated bending.

From the mechanical point of view, the CNT films were found to have appreciable tensile strength which was found on par with CNT materials made by other methods [35-38]. As shown in the Fig. 5a, various CNT films presented slightly different behaviour. The samples of particular predominant chirality were much more brittle than the unsorted ones (NC7000, carpet). The underlying reason is that the $(6,5)$ and $(7,6)$ samples are composed of short singlewall CNTs whereas the unsorted material is mostly multi-wall having significant length. The 
selection of CNT films used in this work offers either high tensile strength (up to $122 \mathrm{MPa}-$ sorted, single-wall) or can be strained to a large extent (up to $2.4 \%$ - unsorted, multi-wall).

Moreover, it is important to stress that the CNT films remain very durable even after the flash annealing as proven by three point bending evaluation of the material (Fig. 5b). We observed initial increase in electrical resistance by up to $4.0 \%$ (Carpet), 3.1\% (NC7000), 1.9\% (7,6 CNTs) and $1.4 \%(6,5 \mathrm{CNTs})$ due to slight change of the microstructure under strain. Individual CNTs and their bundles slide past each other to reach the optimum arrangement in these conditions. However that increases distance between them, what elevates junction resistance, which is manifested by a small decrease in electrical conductivity in the plot.

\section{Conclusions}

In summary, we developed a convenient and rapid method of formation of durable free-standing CNT macroassemblies of highly-defined nanostructure. It was found that the transformation from powder to macroscopic object is easy, fully-scalable, non-destructive and can employ any desired type of CNTs. That is a very important step towards creation and research on new materials, which could not have been formed before due to technical limitations (macroassemblies of chirality-defined CNTs). With current progress in the field of resolution of CNT mixtures into individual components, we believe that this could be the tool to create unique macroscopic architectures and learn much more about their inherent properties. Moreover, they could be a key ingredient in a wide spectrum of applications ranging from optoelectronic emitters [39], shielding materials [40], sensors [41, 42] or drug-delivery systems [43]. 
From the electrical point of view, in this study we have only tested CNTs which showed semiconductive character. Using highly-metallic CNTs as feed could have given much better results in terms of electrical conductivity, but this is currently a matter of further research.

Lastly, cellulose-derived binder was found ideal for dispersion of the material and creation of the CNT films. Not only it is green chemical because of its abundance in nature, but it is also much safer to use [44] than the common surfactants in the nanocarbon field: Sodium dodecylsulfate [45], Sodium dodecylbenzenesulfonate [46] or Triton-X100 [47], which are harmful, cause irritation and pose hazard. Its key advantage from the processing point of view is in its thermal compatibility, which enables its full removal while leaving the microstructure and chemical composition of the CNT film intact.

\section{Acknowledgments}

D.J., M.R. and K.K.K. acknowledge the European Research Council (under the Horizon 2020 Program, ERC grant agreement 641382) and the Royal Society for the financial support. D.J. would also like to thank National Science Center, Poland (under the Polonez program, grant agreement UMO-2015/19/P/ST5/03799) and the European Union's Horizon 2020 research and innovation programme (Marie Skłodowska-Curie grant agreement 665778). 


\section{References}

[1] D. Janas, K.K. Koziol, A review of production methods of carbon nanotube and graphene thin films for electrothermal applications, Nanoscale 6(6) (2014) 3037-3045.

[2] A. Lekawa-Raus, J. Patmore, L. Kurzepa, J. Bulmer, K. Koziol, Electrical Properties of Carbon Nanotube Based Fibers and Their Future Use in Electrical Wiring, Advanced Functional Materials 24(24) (2014) 3661-3682.

[3] R. Rastogi, R. Kaushal, S.K. Tripathi, A.L. Sharma, I. Kaur, L.M. Bharadwaj, Comparative study of carbon nanotube dispersion using surfactants, Journal of Colloid and Interface Science 328(2) (2008) 421-428.

[4] L. Vaisman, H.D. Wagner, G. Marom, The role of surfactants in dispersion of carbon nanotubes, Advances in Colloid and Interface Science 128-130 (2006) 37-46.

[5] P.-C. Ma, N.A. Siddiqui, G. Marom, J.-K. Kim, Dispersion and functionalization of carbon nanotubes for polymer-based nanocomposites: A review, Composites Part A: Applied Science and Manufacturing 41(10) (2010) 1345-1367.

[6] C.A. Mitchell, J.L. Bahr, S. Arepalli, J.M. Tour, R. Krishnamoorti, Dispersion of Functionalized Carbon Nanotubes in Polystyrene, Macromolecules 35(23) (2002) 8825-8830.

[7] N. Behabtu, C.C. Young, D.E. Tsentalovich, O. Kleinerman, X. Wang, A.W.K. Ma, E.A. Bengio, R.F. ter Waarbeek, J.J. de Jong, R.E. Hoogerwerf, S.B. Fairchild, J.B. Ferguson, B. Maruyama, J. Kono, Y. Talmon, Y. Cohen, M.J. Otto, M. Pasquali, Strong, Light, Multifunctional Fibers of Carbon Nanotubes with Ultrahigh Conductivity, Science 339(6116) (2013) 182-186.

[8] B. Vigolo, A. Pénicaud, C. Coulon, C. Sauder, R. Pailler, C. Journet, P. Bernier, P. Poulin, Macroscopic Fibers and Ribbons of Oriented Carbon Nanotubes, Science 290(5495) (2000) 1331-1334.

[9] J.N. Barisci, M. Tahhan, G.G. Wallace, S. Badaire, T. Vaugien, M. Maugey, P. Poulin, Properties of Carbon Nanotube Fibers Spun from DNA-Stabilized Dispersions, Advanced Functional Materials 14(2) (2004) 133-138.

[10] Y. Murakami, S. Chiashi, Y. Miyauchi, M. Hu, M. Ogura, T. Okubo, S. Maruyama, Growth of vertically aligned single-walled carbon nanotube films on quartz substrates and their optical anisotropy, Chemical Physics Letters 385(3-4) (2004) 298-303.

[11] J.W. Jo, J.W. Jung, J.U. Lee, W.H. Jo, Fabrication of Highly Conductive and Transparent Thin Films from Single-Walled Carbon Nanotubes Using a New Non-ionic Surfactant via Spin Coating, ACS Nano 4(9) (2010) 5382-5388.

[12] L. Dongil, H. Hyun Pyo, L. Chul Jin, P. Chan Won, M. Nam Ki, Microfabrication and characterization of spray-coated single-wall carbon nanotube film strain gauges, Nanotechnology 22(45) (2011) 455301.

[13] K. Jiang, Q. Li, S. Fan, Nanotechnology: Spinning continuous carbon nanotube yarns, Nature 419(6909) (2002) 801-801.

[14] A.E. Aliev, J. Oh, M.E. Kozlov, A.A. Kuznetsov, S. Fang, A.F. Fonseca, R. Ovalle, M.D. Lima, M.H. Haque, Y.N. Gartstein, M. Zhang, A.A. Zakhidov, R.H. Baughman, Giant-Stroke, Superelastic Carbon Nanotube Aerogel Muscles, Science 323(5921) (2009) 1575-1578.

[15] Y.L. Li, I.A. Kinloch, A.H. Windle, Direct spinning of carbon nanotube fibers from chemical vapor deposition synthesis, Science 304(5668) (2004) 276-8.

[16] K. Koziol, J. Vilatela, A. Moisala, M. Motta, P. Cunniff, M. Sennett, A. Windle, High-Performance Carbon Nanotube Fiber, Science 318(5858) (2007) 1892-1895.

[17] S. Iijima, Helical microtubules of graphitic carbon, Nature 354(6348) (1991) 56-58.

[18] S. Hong, S. Myung, Nanotube Electronics: A flexible approach to mobility, Nat Nano 2(4) (2007) 207-208.

[19] X. Lu, Z. Chen, Curved Pi-Conjugation, Aromaticity, and the Related Chemistry of Small Fullerenes (<C60) and Single-Walled Carbon Nanotubes, Chemical Reviews 105(10) (2005) 3643-3696.

[20] J.-C. Charlier, X. Blase, S. Roche, Electronic and transport properties of nanotubes, Reviews of Modern Physics 79(2) (2007) 677-732.

[21] E.T. Thostenson, C. Li, T.-W. Chou, Nanocomposites in context, Composites Science and Technology 65(3-4) (2005) 491-516.

[22] E. Pop, D. Mann, Q. Wang, K. Goodson, H. Dai, Thermal Conductance of an Individual Single-Wall Carbon Nanotube above Room Temperature, Nano Letters 6(1) (2006) 96-100. 
[23] M.-F. Yu, O. Lourie, M.J. Dyer, K. Moloni, T.F. Kelly, R.S. Ruoff, Strength and Breaking Mechanism of Multiwalled Carbon Nanotubes Under Tensile Load, Science 287(5453) (2000) 637-640.

[24] M. Popov, M. Kyotani, R.J. Nemanich, Y. Koga, Superhard phase composed of single-wall carbon nanotubes, Physical Review B 65(3) (2002) 033408.

[25] F. Wang, G. Dukovic, L.E. Brus, T.F. Heinz, Time-Resolved Fluorescence of Carbon Nanotubes and Its Implication for Radiative Lifetimes, Physical Review Letters 92(17) (2004) 177401.

[26] C. Fantini, A. Jorio, M. Souza, M.S. Strano, M.S. Dresselhaus, M.A. Pimenta, Optical Transition Energies for Carbon Nanotubes from Resonant Raman Spectroscopy: Environment and Temperature Effects, Physical Review Letters 93(14) (2004) 147406.

[27] Y.A. Kim, H. Muramatsu, T. Hayashi, M. Endo, M. Terrones, M.S. Dresselhaus, Fabrication of HighPurity, Double-Walled Carbon Nanotube Buckypaper, Chemical Vapor Deposition 12(6) (2006) 327-330.

[28] Z. Wang, Z. Liang, B. Wang, C. Zhang, L. Kramer, Processing and property investigation of singlewalled carbon nanotube (SWNT) buckypaper/epoxy resin matrix nanocomposites, Composites Part A:

Applied Science and Manufacturing 35(10) (2004) 1225-1232.

[29] S. Roy, V. Jain, R. Bajpai, P. Ghosh, A.S. Pente, B.P. Singh, D.S. Misra, Formation of Carbon Nanotube Bucky Paper and Feasibility Study for Filtration at the Nano and Molecular Scale, The Journal of Physical Chemistry C 116(35) (2012) 19025-19031.

[30] D. Janas, K. Koziol, Carbon nanotube fibers and films: synthesis, applications and perspectives of the direct-spinning method, Nanoscale (2016).

[31] D. Janas, S.K. Kreft, K.K.K. Koziol, Printing of highly conductive carbon nanotubes fibres from aqueous dispersion, Materials \& Design 116 (2017) 16-20.

[32] L. Zhang, L. Huang, M. Lind, V. Liao, S. O'Brien, Z. Yu, Excitation, Temperature, and Structural Dependence of Second-Order Raman Modes in Single-Wall Carbon Nanotubes, The Journal of Physical Chemistry C 113(37) (2009) 16432-16438.

[33] G. Beyer, Short communication: Carbon nanotubes as flame retardants for polymers, Fire and Materials 26(6) (2002) 291-293.

[34] J.H. Lehman, M. Terrones, E. Mansfield, K.E. Hurst, V. Meunier, Evaluating the characteristics of multiwall carbon nanotubes, Carbon 49(8) (2011) 2581-2602.

[35] J. Zhang, D. Jiang, H.-X. Peng, F. Qin, Enhanced mechanical and electrical properties of carbon nanotube buckypaper by in situ cross-linking, Carbon 63 (2013) 125-132.

[36] J. Che, P. Chen, M.B. Chan-Park, High-strength carbon nanotube buckypaper composites as applied

to free-standing electrodes for supercapacitors, Journal of Materials Chemistry A 1(12) (2013) 4057-4066.

[37] M.F. Arif, S. Kumar, T. Shah, Tunable morphology and its influence on electrical, thermal and mechanical properties of carbon nanostructure-buckypaper, Materials \& Design 101 (2016) 236-244.

[38] J.Y. Oh, S.J. Yang, J.Y. Park, T. Kim, K. Lee, Y.S. Kim, H.N. Han, C.R. Park, Easy Preparation of SelfAssembled High-Density Buckypaper with Enhanced Mechanical Properties, Nano Letters 15(1) (2015) 190-197.

[39] A. Setaro, M. Adeli, M. Glaeske, D. Przyrembel, T. Bisswanger, G. Gordeev, F. Maschietto, A. Faghani, B. Paulus, M. Weinelt, R. Arenal, R. Haag, S. Reich, Preserving n-conjugation in covalently functionalized carbon nanotubes for optoelectronic applications, Nature Communications 8 (2017) 14281. [40] L.-Q. Zhang, B. Yang, J. Teng, J. Lei, D.-X. Yan, G.-J. Zhong, Z.-M. Li, Tunable electromagnetic interference shielding effectiveness via multilayer assembly of regenerated cellulose as the supporting substrate and carbon nanotube/polymer as the functional layer, Journal of Materials Chemistry C (2017). [41] J. Zhou, H. Yu, X. Xu, F. Han, G. Lubineau, Ultrasensitive, Stretchable Strain Sensors Based on Fragmented Carbon Nanotube Papers, ACS Applied Materials \& Interfaces 9(5) (2017) 4835-4842. [42] M.P. Landry, H. Ando, A.Y. Chen, J. Cao, V.I. Kottadiel, L. Chio, D. Yang, J. Dong, T.K. Lu, M.S. Strano, Single-molecule detection of protein efflux from microorganisms using fluorescent single-walled carbon nanotube sensor arrays, Nat Nano advance online publication (2017).

[43] R.M. Bhaskara, S.M. Linker, M. Vögele, J. Köfinger, G. Hummer, Carbon Nanotubes Mediate Fusion of Lipid Vesicles, ACS Nano (2017).

[44] National Center for Biotechnology Information. PubChem Compound Database; CID=24832091, https://pubchem.ncbi.nlm.nih.gov/compound/24832091 (accessed Jan. 25, 2017). 
[45] National Center for Biotechnology Information. PubChem Compound Database; CID=3423265, https://pubchem.ncbi.nlm.nih.gov/compound/3423265 (accessed Jan. 25, 2017).

[46] National Center for Biotechnology Information. PubChem Compound Database; CID=23662403, https://pubchem.ncbi.nlm.nih.gov/compound/23662403 (accessed Jan. 25, 2017).

[47] National Center for Biotechnology Information. PubChem Compound Database; CID=5590, https://pubchem.ncbi.nlm.nih.gov/compound/5590 (accessed Jan. 25, 2017). 